\title{
GEOMETRIC PHASES AND MONODROMY AT SINGULARITIES
}

\author{
Mark S. Alber ${ }^{1}$ and Jerrold E. Marsden ${ }^{2}$ \\ ${ }^{1}$ Department of Mathematics \\ University of Notre Dame \\ Notre Dame, IN 46556, USA \\ ${ }^{2}$ Department of Mathematics \\ University of California \\ Berkeley, CA 94720, USA
}

\section{Introduction}

In $[23,21,14,43]$ and [3] some new (complex) Hamiltonian soliton and homoclinic structures, phase functions and geometric phases for soliton equations were introduced. In particular, phase phenomena caused by the presence of monodromy at singularities in the space of parameters was studied.

Phase functions in the quasi-periodic case have important applications in the theory of evolution equations (where they appear in connection with the Whitham equations). We investigate them in the singular (soliton, umbilic soliton and homoclinic) cases. In this paper we consider the umbilic and homoclinic solutions in this context.

Umbilic soliton solutions and $n$-soliton solutions are both considered as singular points in the moduli spaces of Jacobi varieties. The Hamiltonian structure and asymptotic reduction obtained here can presumably be understood in terms of a regularization of these singularities. Umbilic solutions differ from the usual solitons and homoclinic orbits in that their spectral polynomial (the basic polynomial associated with the Riemann surface) has only positive roots, while they are all negative in the usual case. The umbilic solitons provide a geometric model for homoclinic orbits that approach low dimensional tori instead of homoclinic points as $t \rightarrow \infty$.

The Hamiltonian flow associated with homoclinic orbits introduced by Devaney [17] for 
the C. Neumann problem coincides with the soliton $x$-flow of the KdV equation. This result, together with the isomorphism between different classes of solutions of the C. Neumann, $\mathrm{KdV}$ and Jacobi problems, leads us naturally to the introduction of umbilic homoclinics.

The general approach is demonstrated for the $n$-soliton solutions of the Nonlinear Schrödinger (NLS) and sine-Gordon (SG) equations and umbilic soliton solutions of Kortewegde Vries (KdV) hierarchies of equations.

In the process of investigating the new angle-representations for the homoclinic orbits and soliton-like solutions of the nonlinear equations, we deal in a natural way with the phenomena of geometric phases.

We recall that in [11], Berry considered a geometric phase factor $\exp (i \gamma)$ for systems that are slowly (adiabatically) transported along a closed curve in a space of parameters. In [44] a class of connections was constructed to obtain expressions for the Hannay-Berry phases [13] (these are geometric angle shifts in the classical case) for some integrable problems in terms of the nontrivial holonomy of these connections. In [44], Montgomery gave an example of a phase associated with the presence of singularities in the case of a flat connection. Symmetry and reduction were used to obtain a generalization of geometric phases to the non-integrable case in the form of the holonomy of the Cartan-Hannay-Berry connection ([37]).

All of the papers mentioned above deal with problems that have compact invariant varieties. In [3], we introduced complex angle-representations on associated noncompact Jacobi varieties and use asymptotic reduction to obtain phase functions on the corresponding topologically nontrivial phase spaces. This yields soliton geometric phases and provides a setting for investigating the effect of slow evolution of the solutions of the nonlinear equations in terms of geometric asymptotics (i.e., semiclassical modes).

For the focusing NLS equation, asymptotic reduction of the angle-representation yields a term which remains after the asymptotic splitting of the $n$-soliton solution. This remaining interaction between solitons makes the case of the (f)NLS (focusing nonlinear Schrödinger) equation different from other soliton equations and might have applications in nonlinear optics.

In the context of Hannay-Berry phases, one usually considers a shift of the angle variables $\theta=\left(\theta_{1}, \ldots, \theta_{n}\right)$ in a particular problem after transporting along a closed curve in the space of parameters. This change consists of two parts,

$$
\triangle \theta=\triangle_{D} \theta+\triangle_{G} \theta
$$

called the dynamic and geometric phases. The dynamic phase is due to the dynamical evolution of the system and it is proportional to the period of time $(T)$ during which system is transported along the closed curve. To eliminate the dynamic phase and to retain only the geometric part, averaging is often used. In the soliton case, the period is infinite and so we use the method of "asymptotic reduction" and the complex phase function instead of the averaging approach.

We recall from [3] that there are different approaches to soliton geometric phases.

Our method uses the fact that asymptotic reduction leads to the complex splitting of the spectrum of the soliton problem and yields a new class of geometric phase phenomena 
associated to a moduli of $n$-dimensional noncompact Jacobi varieties. Usually the phase space considered in the context of Hannay-Berry phases is a family of Jacobi tori.

We then show that phases are obtained as a monodromy at singularities of the phase function, which doesn't depend on the initial conditions. A connection between the $\theta$ function and $\tau$-function can also be used to find a link between soliton geometric phases and geometric phases for quasi-periodic solutions (see [5]).

The limiting process and $\tau$-functions on the Jacobi cylinder were investigated by McKean [41] for the KdV equation. The complex geometry related to the $\theta$-functions was previously studied in the KdV case by McKean and Ercolani [41, 22]. The modulational Poisson structure and generalized theta functions $[23,21]$ for the sine-Gordon system were described in terms of conformal ingredients such as differentials on Riemann surfaces and a possible link with the Hamiltonian theory was investigated.

The finite dimensional complex Hamiltonians and phases obtained in this paper can be also used for investigating modulation equations.

Lastly, our soliton Hamiltonian structures lead to presoliton geometric asymptotics and use the results described above to obtain a link with geometric phases in the quantum case.

\section{Umbilic Solitons}

Here we construct a class of soliton-like solutions of the $\mathrm{KdV}$ equation using a singular family of umbilic geodesics on $n$-dimensional quadrics.

It is known $[40,42,51,31,47,7]$ that there are finite dimensional invariant tori in the phase space of completely integrable nonlinear problems and that solutions lying on these tori (hyperelliptic Riemann surface), called quasi-periodic solutions, can be described using a pair of commuting Hamiltonian systems written in configuration variables $\left(\lambda_{1}, \ldots, \lambda_{n}\right)$ and momentum variables $\left(P_{1}, \ldots, P_{n}\right)$.

To obtain the soliton problem, one shrinks pairs of roots of the basic polynomial of the Riemann surface $([41,46,1])$. In [3] we applied the general procidure to the problem of geodesics and make use of the link between the problem of geodesics [8] and the KdV equation. In this paper, we do this for the umbilic case.

Consider a general family of geodesics on the $n$-dimensional ellipsoid

$$
\sum_{j=1}^{n+1} \frac{X_{j}^{2}}{l_{j}}=1
$$

which is described [7] in terms of the root-variables $\left\{\lambda_{j}\right\}$ as a solution of the system of equations

$$
\frac{\partial \lambda_{j}}{\partial x}=\frac{1}{\prod_{i \neq j}\left(\lambda_{j}-\lambda_{i}\right)} \sqrt{\frac{\prod_{k=1, k \neq j_{0}}^{n}\left(\lambda_{j}-m_{k}\right) \prod_{r=1}^{n+1}\left(\lambda_{j}-l_{r}\right)}{\left(-\lambda_{j}\right)}}, \quad j=1, \ldots, n .
$$

To obtain angle-repersentations for different families of umbilic geodesics, we consider the limiting process

$$
m_{k} \rightarrow l_{k+1} \rightarrow b_{k}, \quad k=1, \ldots, n ; \quad k<j_{0},
$$




$$
m_{k} \rightarrow l_{k} \rightarrow b_{k}, \quad k=1, \ldots, n ; \quad k>j_{0},
$$

corresponding to each fixed $\left(1<j_{0}<n\right)$ and transform the system $(2.1)$ as follows

$$
\left.\begin{array}{l}
\sum_{j=1}^{n} \frac{\sqrt{-\lambda_{j}} \lambda_{j}^{\prime}}{\left(\lambda_{j}-b_{k}\right) \sqrt{\left(-\lambda_{j}\right)\left(\lambda_{j}-l_{1}\right)\left(\lambda_{j}-l_{n+1}\right)}}=\frac{\prod_{r=1, r \neq j_{0}, r \neq k}^{n}\left(\lambda_{j}-b_{r}\right)}{\prod_{i \neq j}\left(\lambda_{j}-\lambda_{i}\right)}, \quad k \neq j_{0} . \\
\sum_{j=1}^{n} \frac{\sqrt{-\lambda_{j}} \lambda_{j}^{\prime}}{\sqrt{\left(-\lambda_{j}\right)\left(\lambda_{j}-l_{1}\right)\left(\lambda_{j}-l_{n+1}\right)}}=\frac{\prod_{r=1, r \neq j_{0}}^{n}\left(\lambda_{j}-b_{r}\right)}{\prod_{i \neq j}\left(\lambda_{j}-\lambda_{i}\right)}, \quad k=j_{0} .
\end{array}\right\}
$$

The right hand sides of these expressions are equal to constants

$$
\theta_{k}{ }^{\prime}=0, \quad k=1, \ldots, n, \quad k \neq j_{0} ; \theta_{j_{0}}{ }^{\prime}=1, \quad k=j_{o}
$$

which, after integration, results in the angle-representation

$$
\begin{aligned}
& \theta_{k}=\sum_{j=1}^{n} \int_{\lambda_{j}^{0}}^{\lambda_{j}} \frac{\sqrt{-\lambda_{j}} d \lambda_{j}}{\left(\lambda_{j}-b_{k}\right) \sqrt{\left(\lambda_{j}-l_{1}\right)\left(\lambda_{j}-l_{n+1}\right)}}=\theta_{k}^{0}, \quad k=1, \ldots, n ; \quad k \neq j_{0} . \\
& \theta_{k}=\sum_{j=1}^{n} \int_{\lambda_{j}^{0}}^{\lambda_{j}} \frac{\sqrt{-\lambda_{j}} d \lambda_{j}}{\sqrt{\left(\lambda_{j}-l_{1}\right)\left(\lambda_{j}-l_{n+1}\right)}}=x+\theta_{k}^{0}, \quad k=j_{0} .
\end{aligned}
$$

Theorem 2.1 The system of equations (2.2) of umbilic geodesics on quadrics is a Hamiltonian system with the Hamiltonian

$$
H=\sum_{j=1}^{n} \frac{\left(e^{\left(M\left(\lambda_{j}\right) P_{j}-\left(-\lambda_{j}\right) M^{2}\left(\lambda_{j}\right)\right)}-\prod_{k=1, k \neq j_{0}}^{n}\left(\lambda_{j}-b_{k}\right)\right)}{\left.\prod_{r \neq j}\left(\lambda_{j}-\lambda_{r}\right)\right)}
$$

Here

$$
M(\lambda)=\sqrt{\frac{\left(\lambda-l_{1}\right)\left(\lambda-l_{n+1}\right)}{(-\lambda)}} .
$$

The system (2.5) has a complete set of first integrals

$$
P_{j}=\left(\sum_{k=1, k \neq j_{0}}^{n} \frac{\log \left(\lambda_{j}-b_{k}\right)}{M\left(\lambda_{j}\right)}+\left(-\lambda_{j}\right) M\left(\lambda_{j}\right)\right)
$$

and angle-representation (2.4) that linearize the corresponding Hamiltonian $x$-flow.

Proof Substituting the expressions (2.7) for the integrals into the Hamiltonian system (2.5) we obtain first part of the proof. Then we consider the action-function

$$
S=\sum_{j=1}^{n} \int_{\lambda_{j}^{0}}^{\lambda_{j}} P_{j} d \lambda_{j}
$$


generating a Lagrangian submanifold of the phase space $C^{2 n}$, and consider the following system of variables

$$
\left.\begin{array}{l}
I_{k}=b_{k}, k=1, \ldots, n ; \quad k \neq j_{0} ; I_{j_{0}}=l_{1} l_{n+1} \\
\theta_{k}=-\frac{\partial S}{\partial I_{k}}, k=1, \ldots, n .
\end{array}\right\}
$$

There are no invariant tori in the phase space. Nevertheless, the hamiltonian flow, as we see from (2.4), can be linearized.

Corollary 2.2 Using the isomorphism [8] between the $x$-flow of the problem of geodesics and the $x$-flow of the KdV equation, we obtain the corresponding KdV Hamiltonian. Constructing the $t$-flow on the same invariant variety (2.7), we obtain a new class of soliton-like solutions of the KdV equation that correspond to umbilic geodesics.

Umbilic solitons are described by the system with the following Hamiltonian

$$
H=\sum_{j=1}^{n} \frac{\left(-\sum_{r \neq j} \lambda_{r}+\sum_{k=1, k \neq j_{0}}^{n} b_{k}+l_{1}+l_{n+1}\right)\left(e^{\left(M\left(\lambda_{j}\right) P_{j}-M^{2}\left(\lambda_{j}\right)\right)}-\prod_{k=1, k \neq j_{0}}^{n}\left(\lambda_{j}-b_{k}\right)\right)}{\left.\prod_{r \neq j}\left(\lambda_{j}-\lambda_{r}\right)\right)}
$$

which describes the $t$-flow.

The angle-representation (2.4) has logarithmic singularities. In what follows we will show that it is similar to the soliton (d)NLS (defocusing nonlinear Schrödinger) representations

and reperesentations for the homoclinic orbits of the C. Neumann problem and it can be analyzed using asymptotic reduction (to be described below).

\section{Soliton Hamiltonian Systems and Geometric Phases}

Below we recall from [3] some Hamiltonians and angle-representations for a class of soliton equations. We use a general approach to deduce from the angle-representation, the associated phase functions on associated noncompact Jacobi varieties and we introduce and investigate geometric phases in the complex case.

Now we show that the angle-representation gives the limiting behavior of the $n$-soliton solution and a system of soliton geometric phases. We call the procedure described below the "soliton analysis" and demonstrate it using example of the (d)NLS equation.

\subsection{Defocusing Nonlinear Schrödinger (d)NLS equation}

Using a method similar to the approach of the previous section, it is shown in [3] that soliton solutions of the (d)NLS equation

$$
i \dot{Q}+\frac{1}{2} Q^{\prime \prime}-\bar{Q} Q^{2}=0
$$

and $\mathrm{KdV}$ equation

$$
U_{t}+6 U U_{x}+U_{x x x}=0
$$


can be described by the system with the Hamiltonian

$$
H_{s}^{s}=-\frac{\sum_{j=1}^{n}\left(e^{M\left(\lambda_{j}\right) P_{j}}-\left(\bar{C}\left(\lambda_{j}\right)\right)^{1 / 2} / M\left(\lambda_{j}\right)\right)}{\prod_{r \neq j}\left(\lambda_{j}-\lambda_{r}\right)}
$$

and

$$
H_{s}^{d}=-\frac{\sum_{j=1}^{n}\left(-\sum_{l \neq j} \lambda_{l}-\sum_{k=1}^{n} a_{k}\right)\left(e^{M\left(\lambda_{j}\right) P_{j}}-\left(\bar{C}\left(\lambda_{j}\right)\right)^{1 / 2} / M\left(\lambda_{j}\right)\right)}{\prod_{r \neq j}\left(\lambda_{j}-\lambda_{r}\right)}
$$

and with first integrals

$$
P_{j}=\frac{\sum_{k=1}^{n} \ln \left(\lambda_{j}-a_{k}\right)}{M\left(\lambda_{j}\right)} \quad j=1, \ldots, n
$$

Here

$$
\left.\begin{array}{l}
(d) N L S: \quad M\left(\lambda_{j}\right)=2 i \sqrt{-\left(\lambda_{j}-b_{1}\right)\left(\lambda_{j}-b_{2}\right)} \\
K d V: \quad M\left(\lambda_{j}\right)=2 \sqrt{-\lambda_{j}}
\end{array}\right\}
$$

and $\bar{C}(E)=\left(M(E) \prod_{k=1}^{n}\left(E-a_{k}\right)\right)^{2}$ is a polynomial with constant coefficients. The expression (3.3) can be considered as a constraint for (3.4).

The first integrals (3.5) define a Lagrangian submanifold of the phase space $C^{2 n}$ that has the form of the symmetric product

$$
\left.\Gamma:(\Re \times \ldots \times \Re) / \sigma_{n}\right)
$$

of $n$ copies of the Riemann surface

$$
\Re: \quad P=\frac{\sum_{k=1}^{n} \log \left(\lambda-a_{k}\right)}{M(\lambda)} .
$$

Notice that if we took a formal limit of the Hamiltonian structure, we would obtain a phase space with singularities (i.e., a pinched torus). The Hamiltonian structure obtained here is presumably a regularisation of the pinched torus in an appropriate sense, but we shall not pursue that aspect here.

Introduce the following conjugate variables $\bar{I}_{k}$ and $\bar{\theta}_{k}$

$$
\bar{I}_{k}=a_{k}, \quad \bar{\theta}_{k}=-\frac{\partial S}{\partial \bar{I}_{k}}=-\frac{\partial S}{\partial a_{k}}=\sum_{j=1}^{n} \frac{1}{2} \int_{\lambda_{j}^{o}}^{\lambda_{j}} \frac{d \lambda_{j}}{\left(M\left(\lambda_{j}\right)\left(\lambda_{j}-a_{k}\right)\right)} .
$$

Definition 3.1 We call a complete set of variables $\bar{\theta}_{j}, j=1, \ldots, n$, an angle-representation of the multi-soliton solution on the associated $n$-dimensional complex Lagrangian submanifolds. It describes a map of Abel-Jacobi type determining a noncompact Jacobi variety.

Theorem 3.2 In terms of the variables $\left(\bar{I}_{k}, \bar{\theta}_{k}\right)$, the soliton Hamiltonian flows are linearized

$$
\bar{\theta}_{k}=x+v_{k} t+\varphi_{k}, \quad \bar{I}_{k}=a_{k}, \quad v_{k}=2 a_{k} \quad k=1, \ldots, n
$$

on the noncompact Jacobi variety introduced above. 


\subsection{The Method of Asymptotic Reduction}

We consider the expression for the $n$-soliton angle-variables

$$
\bar{\theta}_{r}=\sum_{j=1}^{n} \frac{1}{2} \int_{\lambda_{j}^{o}}^{\lambda_{j}} \frac{d \lambda_{j}}{\left(M\left(\lambda_{j}\right)\left(\lambda_{j}-a_{r}\right)\right)}=x+2 v_{r} t, \quad r=1, \ldots, n
$$

for particular choice of initial values of the root-variables

$$
\lambda_{j}(0,0)=\lambda_{j}^{o}=\frac{a_{j-1}+a_{j}}{2} .
$$

Note that in the general case, basic points $\lambda_{j}^{o}$ of the angle map (3.9) are different from the initial points $\lambda_{j}(0,0)$.

Note that term $2 v_{r} t$ will generate a dynamical soliton phase

$$
\Delta_{D} \bar{\theta}_{r}=2 v_{r} T .
$$

Here $\mathrm{T}$ is in the context of Hannay-Berry phases a period of time during which system is transported alond a closed curve in the space of parameters. $\Delta_{D} \bar{\theta}_{r}$ is a shift of the angle variables due to dynamics. Averaging of the angle variables is usually used to eliminate this term and to calculate additional (geometric) phase. Our case is special since we are dealing with the infinite period $\mathrm{T}$. Instead of averaging we will use the method of asymptotic reduction

Theorem 3.3 As $(t \rightarrow \infty)$ (or $(t \rightarrow-\infty))$ the system of angle variables of the $n$-soliton solution (3.9) splits into $n$ 1-soliton angle variables. We shall call this process asymptotic reduction.

Proof In what follows we will investigate $(3.11)$ as $(t \rightarrow \infty)($ or $(t \rightarrow-\infty))$. We define a direction in the $(x, t)$ plane by fixing

$$
x+v_{k} t=d_{k}=\text { const }
$$

for some $r=k$. Then we transform the $k$-th equation from (3.11) adding and subtracting integrals along different intervals on the real axis to obtain a complete integral from 0 to $\lambda_{k}$

$$
\left.\begin{array}{l}
\frac{1}{2} \int_{0}^{\lambda_{k}} \frac{d \lambda}{\left(M(\lambda)\left(\lambda-a_{k}\right)\right)}=d_{k}+\frac{1}{2} \sum_{j<k} \int_{\lambda_{j}}^{\lambda_{j+1}^{o}} \frac{d \lambda_{j}}{\left(M\left(\lambda_{j}\right)\left(\lambda_{j}-a_{k}\right)\right)} \\
+\frac{1}{2} \int_{0}^{\lambda_{1}^{o}} \frac{d \lambda_{j}}{\left(M\left(\lambda_{j}\right)\left(\lambda_{j}-a_{k}\right)\right)}-\frac{1}{2} \sum_{j>k} \int_{\lambda_{j}^{o}}^{\lambda_{j}} \frac{d \lambda_{j}}{\left(M\left(\lambda_{j}\right)\left(\lambda_{j}-a_{k}\right)\right)}+\varphi_{k}^{o} .
\end{array}\right\}
$$

Here $\varphi_{k}^{o}$ is a term chosen to be consistent with the initial data (3.12). Notice that in the real case (when all $\lambda_{j}$ and $P_{j}$ are real), every $\lambda_{j}$ is varying once along the cycle $l_{j}$ over the basic cut $\left[a_{j}, a_{j-1}\right]$ on the Riemann surface (3.5) with the $P_{j}$ 's treated as constants. 
The rest of the (3.11) can be described for a fixed $d_{k}$ as follows

$$
\sum_{j=1}^{n} \frac{1}{2} \int_{\lambda_{j}^{o}}^{\lambda_{j}} \frac{d \lambda_{j}}{\left(M\left(\lambda_{j}\right)\left(\lambda_{j}-a_{r}\right)\right)}=x+v_{r} t=d_{k}+\left(v_{r}-v_{k}\right) t, \quad r \neq k, \quad r=1, . ., n .
$$

Here $\left(v_{r}-v_{k}\right)>0, r<k$, and $\left(v_{r}-v_{k}\right)<0, r>k$.

System (3.15) yields the following limits

$$
\left.\begin{array}{l}
\text { 1. } t \rightarrow \infty: \lambda_{r+1} \rightarrow a_{r} \text { for } r>k \quad \text { and } \lambda_{r} \rightarrow a_{r} \text { for } r<k \\
\text { 2. } t \rightarrow-\infty: \lambda_{r} \rightarrow a_{r} \text { for } r>k \text { and } \lambda_{r+1} \rightarrow a_{r} \text { for } r<k .
\end{array}\right\}
$$

This transforms (3.14) into the expression for the 1-soliton angle variable

$$
\bar{\Theta}_{k}=\frac{1}{2} \int_{0}^{\lambda_{k}} \frac{d \lambda}{\left(M(\lambda)\left(\lambda-a_{k}\right)\right)}=x+v_{k} t+\Phi_{k}, \quad k=1, \ldots, n .
$$

Here

$$
\Phi_{k}=\varphi_{k}+\varphi_{k}^{o}
$$

\subsection{Complex Phase Function and Geometric Phases}

Now let us investigate a link between the angle-map obtained above, the Abel-Jacobi map, and geometric phases. There are three approaches: complex splitting of the spectrum, averaging and method of complex phase function, and the $\tau$-function approach.

Definition 3.4 We define soliton geometric phases as follows:

$$
\Delta \varphi=\oint_{C} d_{a}(\varphi)
$$

Here $d_{a}$ and $C$ denote the differential and a closed curve in the space of parameters (a) respectively.

The integral in (3.19) depends on the choice of connection in the space of parameters. For example, in the case of a flat connection, we get the following result.

Theorem 3.5 Some of the soliton geometric phases coincide with the phase shift of the $k$-th soliton of the multi-soliton solution and can be described by the following singular integral

$$
\Delta \varphi_{k}=\frac{1}{2} \oint_{L_{k}} \frac{d \lambda}{\left(M(\lambda)\left(\lambda-a_{k}\right)\right)}, \quad k=1, \ldots, n
$$

Here $L_{k}$ is a cycle over the cut $\left[0, a_{k}\right]$ on the Riemann surface. 
Proof In what follows we consider time $(t)$ as a parameter. A closed curve in $t$-space will be regarded as a curve from $(-\infty)$ to $(\infty)$.

Lemma 3.6 Asymptotic reduction results, in particular, in the splitting of every element of the discrete spectrum $a_{k}$ into a pair of pure imaginary points $\left(-i \alpha_{k}, i \alpha_{k}\right)$. The phase function $\varphi_{k}$ is defined on the covering space of the generalized Jacobian.

In what follows we demonstrate general approach using the (d)NLS equation as an example. We use the change of variables

$$
\xi_{j}^{2}(x)=\frac{\left(\lambda_{j}-b_{1}\right)}{\left(b_{2}-\lambda_{j}\right)}, \quad \alpha_{k}^{2}=\frac{\left(a_{k}-b_{1}\right)}{\left(b_{2}-a_{k}\right)}, \quad \rho_{k}=\sqrt{\left(b_{2}-a_{k}\right)\left(a_{k}-b_{1}\right)} \text { in NLS case }
$$

to formally integrate the basic expression occuring in the right hand side of (3.14)

$$
\int \frac{d \lambda_{j}}{2 M\left(\lambda_{j}\right)\left(\lambda_{j}-a_{k}\right)}=\int \frac{d \xi_{j}}{\left(\left(\xi_{j}\right)^{2}-\left(\alpha_{k}\right)^{2}\right)}=\frac{1}{2 \rho_{k}} \log \left|\frac{\left(\xi_{j}-\alpha_{k}\right)}{\left(\xi_{j}+\alpha_{k}\right)}\right| .
$$

Lastly we obtain (3.20) as a sum of values of the (3.17) for $d_{k} \rightarrow \infty$ and $d_{k} \rightarrow-\infty$. Note that we take integral in (3.17) with different signs since for $d_{k} \rightarrow \infty$ and $d_{k} \rightarrow-\infty$ it is defined on different sheets of the Riemann surface

$$
W^{2}=\frac{1}{M(\lambda)^{2}\left(\lambda-a_{k}\right)^{2}} .
$$

Now, we want to define limit points $\lambda_{j}^{1}\left(\right.$ or $\left.\xi_{j}^{1}\right)$ of the angle-map so that (3.22) and (3.18) are real-valued for every $j=1, \ldots, n$.

There are two choices, which we take for $\left(d_{k} \rightarrow-\infty\right)$ and $\left(d_{k} \rightarrow \infty\right)$. It results in two different relations between the phases of the solitons $\left(\varphi_{k}^{+}, \varphi_{k}^{-}\right)$and the scattering data (parameters of the system), namely the discrete spectrum $a_{j}$. Thus, for the flat connection we get

$$
\Delta \varphi_{k}=\varphi_{k}^{+}-\varphi_{k}^{-}
$$

Therefore, we have obtained a new class of classical geometric phases for the systems defined on associated noncompact Jacobi varieties in the case of infinite period $(\mathrm{T})$.

In what follows we demonstrate our method in complex case using the angle representations for the $n$-soliton solutions of the focusing NLS equation and for the the breather and kink-kink solutions of the sine-Gordon equation.

\subsection{Complex Geometric Phases and Angle-representations}

For the focusing Nonlinear Schrödinger (f)NLS equation

$$
i \dot{Q}+\frac{1}{2} Q^{\prime \prime}+\bar{Q} Q^{2}=0
$$


the differential soliton system has Hamiltonians

$$
H_{s}^{s}=-\frac{\sum_{j=1}^{g}\left(e^{P_{j}}-\bar{C}\left(\lambda_{j}\right)\right)}{\prod_{r \neq j}\left(\lambda_{j}-\lambda_{r}\right)}
$$

and

$$
H_{s}^{d}=-\frac{\sum_{j=1}^{g} D_{j}\left(e^{P_{j}}-\bar{C}\left(\lambda_{j}\right)\right)}{\prod_{r \neq j}\left(\lambda_{j}-\lambda_{r}\right)}
$$

and first integrals

$$
P_{j}=\sum_{k=1}^{g} \log \left(\lambda_{j}-a_{k}\right)\left(\lambda_{j}-\bar{a}_{k}\right) \quad j=1, \ldots, n
$$

Here

$$
D_{j}=2 i\left(-\sum_{l \neq j}^{g} \lambda_{l}+\sum_{k=1}^{N}\left(a_{k}+\bar{a}_{k}\right)\right) .
$$

Hamiltonian (3.26) is defined on the cotangent bundle $T^{*}\left((\Re \times \ldots \times \Re) / \sigma_{n}\right)$ where the Riemann surface has the form

$$
\Re: P=\sum_{k=1}^{g} \log \left(\lambda-a_{k}\right)\left(\lambda-\bar{a}_{k}\right)
$$

Complex (NLS) angle-reperesentation can be described as follows

$$
\theta_{r}=-\operatorname{Im}\left(a_{k}\right) \sum_{j=1}^{g} \int_{\lambda_{j}^{o}}^{\lambda_{j}}\left(\frac{1}{\left(\lambda_{j}-a_{r}\right)}-\frac{1}{\left(\lambda_{j}-\bar{a}_{r}\right)}\right) d \lambda_{j}=x+v_{r} t, \quad r=1, \ldots, N,
$$

and

$$
\theta_{r+N}=\frac{a_{r}-a_{r+1}}{2 i} \sum_{j=1}^{g} \int_{\lambda_{j}^{o}}^{\lambda_{j}}\left(\frac{1}{\left(\lambda_{j}-a_{r}\right)}-\frac{1}{\left(\lambda_{j}-\bar{a}_{r+1}\right)}\right) d \lambda_{j}=x+v_{r} t, \quad r=1, \ldots, N-1 .
$$

Here

$$
v_{r}=2 \operatorname{Re}\left(a_{r}\right), \quad r=1, \ldots, N ; \quad v_{r}=a_{r}+\bar{a}_{r+1}, \quad r=N+1, \ldots, 2 N-1 .
$$

Now we take real parts of the expressions (3.30) and (3.31)

$$
\begin{gathered}
\operatorname{Re}\left(\theta_{r}\right)=d_{k}+\left(v_{r}-v_{k}\right) t, \quad r \neq k, r=1, \ldots, N, \\
\operatorname{Re}\left(\theta_{r+N}\right)=d_{k}+\frac{\left(\left(v_{r}-v_{k}\right)+\left(v_{r+1}-v_{k}\right)\right)}{2} t, \quad r \neq k, r=1, \ldots, N-1 .
\end{gathered}
$$

and apply asymptotic reduction along direction $d_{k}(1 \leq k \leq N)$ in a way described in $\S 3$. It yields the following limits for the root variables

$$
\left.\begin{array}{rl}
\text { 1. } t \rightarrow \infty: & \lambda_{r+1} \rightarrow \bar{a}_{r}, \lambda_{r+N} \rightarrow a_{r} \text { for } r>k, \\
& \lambda_{r} \rightarrow a_{r}, \lambda_{r} \rightarrow a_{r} \text { for } r<k \\
\text { 2. } t \rightarrow-\infty: & \lambda_{r} \rightarrow a_{r} \text { for } r>k \text { and } \lambda_{r+1} \rightarrow a_{r} \text { for } r<k .
\end{array}\right\}
$$


This yields the spliting of (3.30) into $N$ 1-soliton angle representations with corresponding phase functions. Therefore, the angle representation (3.30) describes $N$-soliton solutions.

The equations (3.31) describe the interaction between 1-solitons which remains after the asymptotic splitting of the $n$-soliton solution. It makes the case of (f)NLS equation different from other soliton equations and might have important applications in nonlinear optics.

To deal with the limit of the argument part of (3.30), we return to the the formulae connecting $Q$ to solutions $U$ of the (NLS)

$$
U=i \frac{\partial}{\partial x} \log Q
$$

Integrating this expression and considering limit (3.35) we obtain arbitrary constant in the phase of the exponent. We put this constant equal to the argument part of the phase function corresponding to the limit (3.35).

The soliton system for the sine-Gordon equation (SGE)

$$
U_{x x}-U_{t t}=\sin U
$$

has the following Hamiltonians

$$
\begin{aligned}
& H_{s}^{s}=-2 \frac{\sum_{j=1}^{n}\left(e^{\sqrt{-\lambda_{j}} P_{j}}-\bar{C}\left(\lambda_{j}\right)\right)\left(1+G_{N-1}\left(\lambda_{j}\right)\right)}{\prod_{r \neq j}\left(\lambda_{j}-\lambda_{r}\right)} . \\
& H_{s}^{d}=-2 \frac{\sum_{j=1}^{n}\left(e^{\sqrt{-\lambda_{j}} P_{j}}-\bar{C}\left(\lambda_{j}\right)\right)\left(1-G_{N-1}\left(\lambda_{j}\right)\right)}{\prod_{r \neq j}\left(\lambda_{j}-\lambda_{r}\right)} .
\end{aligned}
$$

and complete system of first integrals

$$
P_{j}=\frac{\log \bar{C}\left(\lambda_{j}\right)}{\sqrt{-\lambda_{j}}}, \quad j=1, \ldots, N
$$

which has a system of action-angle variables. Here $n=n_{1}$ or $n=n_{2}$.

Corresponding action-angle variables can be described as follows in kink-kink case:

$$
I_{k}=a_{k}, \quad \theta_{k}=-\frac{\partial S}{\partial I_{k}}=\sum_{j=1}^{n_{1}} \int_{\lambda_{j}^{o}}^{\lambda_{j}} \frac{1}{\left(\lambda_{j}-a_{k}\right)} \frac{d \lambda_{j}}{\sqrt{-\lambda_{j}}}, \quad k=1, \ldots, n_{1},
$$

in breather case:

$$
I_{k}=a_{k}, \quad \theta_{k}=\sum_{j=1}^{n_{2}} \int_{\lambda_{j}^{o}}^{\lambda_{j}}\left(\frac{1}{\left(\lambda_{j}-a_{k}\right)} \frac{d \lambda_{j}}{\sqrt{-\lambda_{j}}}=w_{k} x+v_{k} t, \quad k=1, . ., n_{2},\right.
$$




$$
I_{k+n_{2}}=\bar{a}_{k}, \quad \theta_{k+n_{2}}=\sum_{j=1}^{n_{2}} \int_{\lambda_{j}^{o}}^{\lambda_{j}}\left(\frac{1}{\left(\lambda_{j}-\bar{a}_{k}\right)} \frac{d \lambda_{j}}{\sqrt{-\lambda_{j}}}=\bar{w}_{k} x+\bar{v}_{k} t, \quad k=1, . ., n_{2} .\right.
$$

Here

$$
w_{k}=\left(1-\frac{1}{4 a_{k}}\right), \quad v_{k}=\left(1+\frac{1}{4 a_{k}}\right)
$$

The soliton (kink-kink) angle representation for the sine Gordon equation is equivalent to a particular case of the KdV and (d)NLS representation $\left(n_{1}=2 n_{2}\right)$.

The soliton (breather) angle representation for the sine-Gordon equation is transformed into kink-kink representation if one takes $\left(n_{1}=2 n_{2}\right)$ and $\left(a_{2 k}, a_{2 k+1}\right) \rightarrow b_{k}, k=1, \ldots, n_{2}$, and then $b_{k} \rightarrow\left(a_{k}, \bar{a}_{k}\right)$.

We finally combine (3.41) and (3.42) to obtain angle representation for the $\left(N_{1}+N_{2}\right)$ - soliton solution of the sine Gordon equation.

Investigating this representation one obtains corresponding geometric phases.

\section{Umbilic Soliton Angle-Representations}

From the results of $\S 2$, we see that the angle-representation for umbilic solitons is similar to the case of "dark-hole" solitons of the (d)NLS equation. Asymtotic reduction for the umbilic solitons defined by the system with the Hamiltonian (2.9) can be described as follows.

Note that angle-representation corresponding to (2.9) has the form

$$
\begin{aligned}
& \theta_{k}=\sum_{j=1}^{n} \int_{\lambda_{j}^{0}}^{\lambda_{j}} \frac{\lambda_{j} \sqrt{\lambda_{j}} d \lambda_{j}}{\left(\lambda_{j}-b_{k}\right) \sqrt{\left.\left(l_{1}\right)-\lambda_{j}\right)\left(\lambda_{j}-l_{n+1}\right)}}=\theta_{k}^{0}+x+v_{k} t, \quad k=1, \ldots, n ; \quad k \neq j_{0} . \\
& \theta_{j_{0}}=\sum_{j=1}^{n} \int_{\lambda_{j}^{0}}^{\lambda_{j}} \frac{\sqrt{\lambda_{j}} d \lambda_{j}}{\sqrt{\left.\left(l_{1}\right)-\lambda_{j}\right)\left(\lambda_{j}-l_{n+1}\right)}}=\theta_{j_{0}}^{0}+x+v_{j_{0}} t .
\end{aligned}
$$

Now let us fix the direction $d_{j_{0}}$ :

$$
d_{j_{0}}=x+v_{j_{0}} t=\text { const. }
$$

and consider what happens with other elements of the angle-representation as $t \rightarrow \infty$

$$
\theta_{k}=d_{j_{0}}+\left(v_{k}-v_{j_{0}}\right) t
$$

An analysis of this process results in the following limiting values of $\lambda_{k}$

$$
\lambda_{k} \rightarrow b_{k}, \quad k=1, \ldots, n, \quad k \neq j_{0} .
$$

Substituting these limiting values in the expression (4.1), one obtains

$$
\theta_{j_{0}}=\sum_{k=1, k \neq j_{0}}^{n} \int_{\lambda_{k}^{0}}^{b_{k}} \frac{\sqrt{\lambda_{k}} d \lambda_{k}}{\sqrt{\left.\left(l_{1}\right)-\lambda_{j}\right)\left(\lambda_{j}-l_{n+1}\right)}}+\int_{\lambda_{j_{0}}^{0}}^{\lambda_{j_{0}}} \frac{\sqrt{\lambda_{j_{0}}} d \lambda_{j_{0}}}{\sqrt{\left.\left(l_{1}\right)-\lambda_{j}\right)\left(\lambda_{j}-l_{n+1}\right)}}=d_{j_{0}}
$$


meaning that the initial system (4.1) is reduced asymptotically to the one dimensional periodic solution

$$
\Theta_{j_{0}}=\int_{\lambda_{j_{0}}^{0}}^{\lambda_{j_{0}}} \frac{\sqrt{\lambda_{j_{0}}} d \lambda_{j_{0}}}{\sqrt{\left.\left(l_{1}\right)-\lambda_{j}\right)\left(\lambda_{j}-l_{n+1}\right)}}=\Theta_{0}^{0}+x+v_{j_{0}} t .
$$

Now we apply our method for investigating so-called "solitons with a quasi-periodic background". The corresponding angle-representation consists of two different parts and it describes an interaction between solitons and quasi-periodic solutions.

The general limiting process leading to the soliton solutions of this type can be described as follows. Let

$$
C(\lambda)=\prod_{k=1}^{2 n+1}\left(\lambda-m_{k}\right)
$$

denote basic polynomial of the Riemann surface for the quasi-periodic solutions of the $\mathrm{KdV}$ equation. Then general limit process leading to the "solitons with a quasi-periodic background" can be described as follows:

$$
\left[m_{2 k_{r}}, m_{2 k_{r}+1}\right] \rightarrow b_{k_{r}} .
$$

Here

$$
k_{r} \in\left(k_{1}, \ldots, k_{d}\right), \quad 1 \leq k_{r} \leq n, \quad 1 \leq d \leq(n-1) .
$$

This yields the corresponding angle-representation

$$
\left.\begin{array}{l}
\theta_{r}=\sum_{j=1}^{n} \int_{\lambda_{j}^{0}}^{\lambda_{j}} \frac{\lambda_{j} d \lambda_{j}}{\left(\lambda_{j}-b_{k_{r}}\right) \sqrt{\prod_{m_{s} \in M}\left(\lambda_{j}-m_{s}\right)}}=\theta_{r}^{0}+x+v_{r} t, \quad r=1, \ldots, d \\
\theta_{r}=\sum_{j=1}^{n} \int_{\lambda_{j}^{0}}^{\lambda_{j}} \frac{\lambda_{j}^{n-r} d \lambda_{j}}{\sqrt{\prod_{m_{s} \in M}\left(\lambda_{j}-m_{s}\right)}}=\theta_{r}^{0}+x+v_{r} t, \quad r=(d+1), \ldots, n
\end{array}\right\}
$$

where

$$
M=\left(m_{s} / 1 \leq s \leq(2 n+1) ; s \neq\left(2 k_{r}\right), s \neq\left(2 k_{r}+1\right)\right), r=1, \ldots, d
$$

Asymptotic reduction of the angle-representation leads to the description of an interaction between solitons and quasi-periodic solutions.

\section{Homoclinic Hamiltonian Systems}

Here we show that Hamiltonian flows of the homoclinic orbits described by Devaney [17] for the C. Neumann problem coincide with the soliton Hamiltonian $x$-flows for the KdV equation. This is important since it gives an understanding of the link between soliton solutions and homoclinic orbits for associated completely integrable systems.

It enables one to treat the homoclinic case in the same manner as the soliton case and to introduce homoclinic Hamiltonians, angle-repersentations, and geometric phases. In particular, using the Knörrer-Moser isomorphism between the Jacobi problem of geodesics and 
the C. Neumann problem, we introduce new orbits corresponding to the umbilic geodesics and give a geometric interpretation of the homoclinic orbits of Devaney.

\subsection{Homoclinics and Solitons}

First of all, we note that a system of first integrals inrtoduced by Devaney and Uhlenbeck for the C. Neumann problem, namely

$$
\begin{gathered}
\Phi_{j}(y, \dot{y}): T S^{n} \rightarrow R, \\
\Phi_{j}(y, \dot{y})=y_{j}^{2}+\frac{1}{2} \sum_{k \neq j} \frac{\left(\dot{y}_{j} y_{k}-\dot{y}_{k} y_{j}\right)^{2}}{l_{k}^{2}-l_{j}^{2}}, j=1, \ldots, n
\end{gathered}
$$

play central role in Devaney's description of the transversal homoclinic orbits. Here $l_{0}<l_{1}<l_{0}<\ldots<l_{n}$ and $l_{0}=0$. Namely, he proved the following Lemma.

Lemma 5.1 The first integrals are identically zero along the orbits; i.e.,

$$
\Phi_{j}(y, \dot{y})=0, j=1, \ldots, n .
$$

We use this fact together with the algebraic-geometric description of the quasi-periodic solutions $[8,9]$ of the C. Neumann problem to obtain the following result.

Theorem 5.2 The Hamiltonian flow of the homoclinic orbits of the C. Neumann problem coincide with the soliton Hamiltonian $x$-flow of the KdV equation. Both flows are defined on a noncompact Jacobi variety.

Proof In [8, 9], quasi-periodic solutions of the C. Neumann problem were discribed using root-variables $\lambda_{j}$, i.e., solutions of the system

$$
\frac{\partial \lambda_{j}}{\partial x}=\frac{\sqrt{-\prod_{r=1}^{n}\left(\lambda_{j}-m_{r}\right) \prod_{k=1}^{n}\left(\lambda_{j}-a_{k}\right)}}{\prod_{i \neq j}\left(\lambda_{j}-\lambda_{i}\right)}, \quad j=1, \ldots, n
$$

and action-angle variables defined on the Jacobi variety of the symmetric product

$$
\Gamma:\left((\Re \times \ldots \times \Re) / \sigma_{n}\right)
$$

of the $\mathrm{n}$ copies of the Riemannian surface

$$
\Re: \quad P^{2}=-\prod_{k=1}^{n+1}\left(\lambda-a_{k}\right) \prod_{r=1}^{n}\left(\lambda-m_{r}\right),
$$

$\Gamma$ being a Lagrangian submanifold of the phase space $C^{2 n}$.

In this setting, the first integrals of the problem can be represented in the form

$$
\Phi_{j}=F_{j}=\frac{\prod_{r=1}^{n}\left(a_{j}-m_{r}\right)}{\prod_{k \neq j}\left(a_{j}-a_{k}\right)} .
$$


Here

$$
a_{j}=-2 l_{j}^{2}, \quad j=0, \ldots, n .
$$

Condition (5.3) and formula (5.7) yield the following choice of first integrals $m_{j}$

$$
m_{j}=a_{j}, j=1, \ldots, n ; a_{0}=0,
$$

meaning that all roots of the basic polynomial of the Riemannian surface (5.6) are double negative roots except $a_{0}=0$.

Therefore, the system (5.4) corresponding to the case of a singular spectrum (5.8) coincides precisely with the system of equations describing soliton solutions of the KdV equation. It leads to the introduction of the exponential homoclinic Hamiltonians with logarithmic first integrals and angle-representation

$$
\theta_{r}=\sum_{j=1}^{n} \frac{1}{2} \int_{\lambda_{j}^{o}}^{\lambda_{j}} \frac{d \lambda_{j}}{\left.\sqrt{-\lambda_{j}}\left(\lambda_{j}-a_{r}\right)\right)}=x+\theta_{r}^{0}, \quad r=1, \ldots, n
$$

defined on the noncompact Jacobi variety of the symmetric product of $n$ copies of the Riemannian surface

$$
P=\frac{\log \left(\lambda-a_{r}\right)}{\sqrt{-\lambda}}
$$

Corollary 5.3 As $(x \rightarrow \infty$ ) (or $x \rightarrow-\infty$ ), the spectrum of the homoclinic orbit splits into complex pairs

$$
a_{j} \rightarrow\left(i \alpha_{j},-i \alpha_{j}\right), \quad a_{j}=-\alpha_{j}^{2}, \quad j=1, \ldots, n
$$

and an analysis of the angle representation yields an introduction of the following homoclinic point $(p)$

$$
\lambda_{j}=a_{j}, \quad j=1, \ldots, n
$$

with corresponding stable $W^{s}$ (and ustable $W^{u}$ ) manifolds, which consist of the orbits on the two different sheets of the double covering of the Riemannian surface (5.10) defined by the following change of variables

$$
\xi_{j}^{2}=-\lambda_{j}, \quad j=1, \ldots, n .
$$

Orbits on the stable and ustable manifolds are forward (and backward) asymptotic to ( $p$ )

$$
\xi_{j} \rightarrow \alpha_{j}, \quad j=1, \ldots, n
$$

and

$$
\xi_{j} \rightarrow-\alpha_{j}, \quad j=1, \ldots, n
$$

respectively. 
The splitting of the spectrum follows from evaluating the basic integral from (5.9)

$$
\frac{1}{2 \alpha_{j}} \log \left(\frac{\xi_{j}-\alpha_{j}}{\xi_{j}+\alpha_{j}}\right)
$$

and analysing the angle-reperesentation (5.9) as $x \rightarrow \infty$ (or $\mathrm{x} \rightarrow-\infty$ ).

Lastly, we define another Hamiltonian flow on the invariant variety of the homoclinic orbit and obtain exactly the soliton KdV problem.

An interesting example of the (SG) homoclinic solution in the two-dimensional case was considered in $[15,26]$, where special real action-angle variables were used to describe a breather-kink-antikink transformation through a homoclinic orbit.

We introduce below an angle-representations for such orbits in the $n$-dimensional case.

Theorem 5.4 Soliton (kink-kink) and breather angle representations for the sine Gordon equation are separated by the following singular class of "resonant solutions"

$$
\begin{gathered}
\theta_{k}=\sum_{j=1}^{n_{1}} \int_{\lambda_{j}^{o}}^{\lambda_{j}} \frac{1}{\left(\lambda_{j}-b_{k}\right)} \frac{d \lambda_{j}}{\sqrt{-\lambda_{j}}}, \quad k=1, \ldots, n_{2} . \\
\theta_{k+n_{2}}=\sum_{j=1}^{n_{1}} \int_{\lambda_{j}^{o}}^{\lambda_{j}} \frac{1}{\left(\lambda_{j}-b_{k}\right)^{2}} \frac{d \lambda_{j}}{\sqrt{-\lambda_{j}}}, \quad k=1, \ldots, n_{2} .
\end{gathered}
$$

obtained from (3.42).

Note also that the phase function can be obtained using the fact that

$$
\theta_{k+n_{2}}=\frac{\partial \theta_{k}}{\partial b_{k}}
$$

Here $b_{k}, \quad k=1, \ldots, n_{2}$ are negative real numbers and $N_{1}=2 n_{1}=4 n_{2}$.

Therefore, the homoclinic orbit in this case is a separatrix solution associated with a degenerate system of action variables and a nondegenerate angle-representation.

\subsection{Geometry of the Homoclinic Orbits}

The isomorphism between classes of solutions of Jacobi problem and those of the C.Neumann problem can be used in the opposite direction. Namely, one can consider a special family of umbilic geodesics on the $n$-dimensional hyperbolloids corresponding to the homoclinic orbits.

In what follows, we use the isomorphism mentioned above in the form established in $[9,8]$

$$
z_{j}^{2}=\frac{\frac{x_{j}}{d_{j}}}{\sum_{k=1}^{n} \frac{x_{j}^{2}}{d_{j}}}, \quad a_{j}=\frac{1}{d_{j}}, \quad \lambda_{j}=\frac{1}{\eta_{j}}, \quad j=1, \ldots, n,
$$

which relates parameters and variables of the two systems. Here $z_{j}$ and $\eta_{j}$ denote variables of the problem of geodesics and $d_{j}$ are semiaxes of the corresponding quadrics. This change of variables (5.19) enables one to avoid Abeleian integrals of the second type in the anglerepresentation. 
In our case all $a_{j}<0$ but $a_{0}=0$; therefore geodesics which correspond to the homoclinic orbits form a singular family of geodesics on a hyperbolic cylinder with semiaxes $d_{j}<0$ and $d_{0} \rightarrow \infty$.

\section{Soliton Geometric Asymptotics and Geometric Phases}

Another application of the machinery developed here is to geometric asymptotics. In particular, the quantum and classical phases are linked.

Recently, Lagrangian manifolds with complex germs and complex geometric asymptotics have been studied $[19,53,33,4,5]$ in connection with algebraic-geometric methods for nonlinear problems. In particular, quantization using the Maslov method of canonical operators [39] was used [19] for the semiclassical approximation of finite-gap solutions of the Toda lattice and to obtain asymptotics of the eigenfunctions of the quantum periodic Toda chain over a solvable Lie algebras [53].

In $[4,5]$ a method was suggested for constructing local semiclassical solutions (modes) in the form of functions of several complex variables on Jacobian varieties of compact multisheeted Riemannian surfaces.

In what follows, we study the quantization of systems with Hamiltonians nonquadratic in $P$ (see the exponential soliton Hamiltonians above). The work here suggests that this can be done on the level of geometric asymptotics and phases. We demonstrate our approach using example of soliton solutions of the KdV equation.

To obtain soliton geometric asymptotics, we first consider the quantum problem corresponding to the finite gap (quasi-periodic) Hamiltonians $[7,4,6]$ for the KdV equation

$$
H^{s}=-\sum_{j=1}^{n} \frac{\left(P_{j}^{2}+C_{2 n+1}\left(\lambda_{j}\right)\right)}{\prod_{r \neq j}\left(\lambda_{j}-\lambda_{r}\right)}
$$

and

$$
H^{d}=-\sum_{j=1}^{n} \frac{2\left(-\sum_{l \neq j} \lambda_{l}-\sum_{k=1}^{2 n+1} m_{k}\right)\left(P_{j}^{2}+C_{2 n+1}\left(\lambda_{j}\right)\right)}{\prod_{r \neq j}\left(\lambda_{j}-\lambda_{r}\right)}
$$

These are quadratic Hamiltonians

$$
H=\frac{1}{2} \sum_{j=1}^{n} g^{j j} P_{j}^{2}+V\left(\lambda_{1}, \ldots, \lambda_{n}\right)
$$

defined on the $T^{*}\left((\Re \times \ldots \times \Re) / \sigma_{n}\right)$. (Here $\Re$ is the Riemann surface: $\left.P^{2}=-C_{2 n+1}(\lambda)\right)$. In accordance with [4] we use the functions $g^{j j}$

$$
g^{j j}=-\frac{1}{\prod_{r \neq j}\left(\lambda_{j}-\lambda_{r}\right)}
$$

and

$$
g^{j j}=-\frac{\left(-\sum_{l \neq j} \lambda_{l}-\sum_{k=1}^{2 n+1} m_{k}\right)}{\prod_{r \neq j}\left(\lambda_{j}-\lambda_{r}\right)} .
$$


as components of the Riemannian metric and construct an operator of Laplace-Beltrami type and then the stationary Schrödinger equation

$$
\nabla^{j} \nabla_{j} U+w^{2}(E-V) U=0
$$

defined on the $n$-dimensional Riemannian manifold. (Here $\nabla^{j}$ and $\nabla_{j}$ are covariant and contravariant derivatives defined by the tensor $g^{j j}$ and $w$ and E are parameters).

Theorem 6.1 The quantum equation (6.6) corresponding to the Hamiltonian (6.1) (or (6.2)) can be reduced to a differential system of $n$ equations

$$
-\frac{1}{w^{2} U_{j}}\left(\frac{\partial^{2} U_{j}}{\partial \lambda_{j}^{2}}+w^{2} C_{2 n+1}\left(\lambda_{j}\right) U_{j}\right)=R_{j}\left(\lambda_{j}\right) \quad j=1, \ldots, n .
$$

connected with each other by means of the symmetry condition

$$
\sum_{j=1}^{n} \frac{R_{j}\left(\lambda_{j}\right) G_{j}}{\prod_{r \neq j}\left(\lambda_{j}-\lambda_{r}\right)}=-E
$$

Here $G_{j}=1$ and $G_{j}=\left(-\sum_{l \neq j} \lambda_{l}-\sum_{k=1}^{2 n+1} m_{k}\right)$ in the cases (6.4) and (6.5) respectively.

Here $R_{j}(\lambda)$ are the same polynomial

$$
R_{j}(\lambda)=R(\lambda)=w_{1} \lambda^{n-1}+w_{2} \lambda^{n-2}+\ldots .+w_{n}, \quad w_{1}=-E
$$

with constant coefficients.

Now we establish a link between equation (6.6) and the initial nonlinear problem by means of geometric asymptotics. Let

$$
\begin{gathered}
U=\sum_{r} A_{r}\left(\lambda_{1}, \ldots, \lambda_{n}\right) \exp \left[i w S_{r}\left(\lambda_{1}, \ldots, \lambda_{n}\right)\right] \\
=\sum_{r} \prod_{j=1}^{n} U_{r j}\left(\lambda_{j}\right)=\sum_{r} \prod_{j=1}^{n}\left(A_{j}\left(\lambda_{j}\right) \exp \left[i w S_{r j}\left(\lambda_{j}\right)\right]\right),
\end{gathered}
$$

which is a function of several complex variables. Substituting (6.10) in (6.7), equating coefficients for $w$ and $w^{2}$ and integrating, we obtain the system of equations

$$
\begin{gathered}
A_{j}^{2}=\frac{c}{\frac{\partial S^{o}}{\partial \lambda_{j}}}=\frac{c}{\sqrt{C_{2 n+1}\left(\lambda_{j}\right)}}, \quad c=\text { const } \\
S^{o}=\sum_{j=1}^{n} S_{j}^{o}\left(\lambda_{j}\right)=\sum_{j=1}^{n} \int_{\lambda_{j}^{o}}^{\lambda_{j}} \sqrt{C_{2 n+1}\left(\lambda_{j}\right)} d \lambda_{j}, \quad j=1, \ldots, n
\end{gathered}
$$

which result in the following form of geometric asymptotics

$$
U=\sum_{r} \frac{A_{o}}{\prod_{j=1}^{n}\left(C_{2 n+1}\left(\lambda_{j}\right)\right)^{\frac{1}{4}}} \exp \left[i w \sum_{j=1}^{n} \int_{\lambda_{j}^{o}}^{\lambda_{j}} \sqrt{C_{2 n+1}\left(\lambda_{j}\right)} d \lambda_{j}\right] .
$$


Let us consider the special class of quasi-periodic solutions of the initial KdV equation defined by the following choice of basic polynomial

$$
C_{2 n+1}(\lambda)=(-\lambda) \prod_{k=1}^{n}\left(\lambda-a_{k}\right)^{2}+R(\lambda)
$$

which depends on exactly $2 \mathrm{n}$ parameters $\left(a_{r}, w_{k}\right)$.

Definition 6.2 We call solutions from this class presoliton solutions.

Theorem $6.3 n$-soliton solutions correspond to the case when all coefficients $w_{k}$ of the polynomial $R(\lambda)$ from (6.14) are equal to zero.

Corollary 6.4 If $R(\lambda)=0$, then formulae (6.13) describes soliton geometric asymptotics with

$$
U=\sum_{l} \frac{A_{o}}{\prod_{j=1}^{n}\left(\sqrt{-\lambda_{j}} \prod_{r=1}^{n}\left(\lambda_{j}-a_{r}\right)\right.} \exp \left[i w \sum_{j=1}^{n} \int_{\lambda_{j}^{o}}^{\lambda_{j}} \sqrt{-\lambda_{j}} \prod_{r=1}^{n}\left(\lambda_{j}-a_{r}\right) d \lambda_{j}\right] .
$$

Lastly, investigating the dependence of soliton geometric asymptotics (6.15) on the slowly changing parameters $a_{l}$, one can also obtain geometric phases in the quantum case. Similar results can be obtained in case of (NLS) and (SG) hierarchies of equations.

In conclusion, we note that geometric asymptotics constructed in this section provide a setting for investigating the semiclassical theory of modulation equations.

\section{References}

[1] M.J. Ablowitz and H. Segur, Solitons and the Inverse Scattering Transform (SIAM, Philadelphia,1981)

[2] M. Adler and P. Van Moerbeke, Completely integrable systems, Kac-Moody Lie algebras and curves, Adv. in Math. 38(1980)267-317

[3] M.S. Alber and J.E.Marsden, On Geometric Phases for Soliton Equations, (preprint)

[4] M.S. Alber, On integrable systems and semiclassical solutions of the stationary Schrödinger equations, Inverse Problems 5 (1989)131-148

[5] M.S. Alber, Complex Geometric Asymptotics, Geometric Phases and Nonlinear Integrable Systems, (to appear)

[6] M.S. Alber, Hyperbolic Geometric Asymptotics, Asymptotic Analysis 5(1991)161-172

[7] M.S. Alber and S.J. Alber, Hamiltonian formalism for finite-zone solutions of integrable equations, C.R. Acad. Sc. Paris 301(1985)777-781 
[8] M.S. Alber and S.J. Alber, Hamiltonian formalism for nonlinear Schrődinger equations and sine-Gordon equations, J. London Math. Soc.36(1987)176-192

[9] S.J. Alber, On Stationary Problems for Equations of Korteweg-de Vries Type, Comm. Pure Appl. Math., 34(1981)259-272

[10] V.I. Arnold, A remark on the branching of hyperelliptic integrals as functions of the parameters, Func. Anal. Appl. 2(1968)187-189

[11] M.V. Berry, Quantal phase factors accompanying adiabatic changes, Proc. R. Soc. Lond. A392(1984)45

[12] M.V. Berry, Classical adiabatic angles and quantal adiabatic phase, J. Phys. A: Math. Gen. 18(1985)15

[13] M.V. Berry and J.H. Hannay, Classical non-adiabatic angles, J. Phys. A: Math. Gen. 21,(1988)325

[14] M.V. Berry, Quantum Adiabatic Anholonomy, Lectures given at the Ferrara School of Theoretical Physics on "Anomalies, defects, phases ...", June 1989, (to be published by Bibliopolis (Naples))

[15] A.R. Bishop, D.W. McLaughlin and M. Solerno, Global coordinates for the breatherkink (antikink) sine-Gordon phase space: An explicit separatrix as a possible source of chaos, Phys. Rev. A, 40(1989)6463-6469

[16] P. Deift, L.C. Li and C. Tomei, Matrix Factorizations and Integrable Systems, Comm. Pure Appl. Math. (1989)443-521

[17] R. Devaney, Transversal Homoclinic Orbits in an Integrable System, Amer. J of Math.,100(1978)631-642

[18] S.U.Dobrohotov and V.P.Maslov, in: S.P.Novikov,ed., Soviet Sciences Reviews,Math.Phys.Reviews, Multiphase asymptotics of nonlinear partial differential equations with a small parameter, (Over. Pub. Ass., Amsterdam, 1982)

[19] S.U. Dobrohotov and V.P. Maslov, Finite-zone, Almost-Periodic Solutions in WKB Approximation ,J. Soviet Math.,16(1981) 1433-1485

[20] H.J. Duistermaat, On global Action-Angle Coordinates, Comm. Pure Appl. Math. 23(1980)687

[21] N. Ercolani, Generalized Theta Functions and Homoclinic Varieties, Proc. Symp. Pure Appl. Math., 49(1989)87-100

[22] N. Ercolani and H.P. McKean, Geometry of $\mathrm{KdV}(4)$ : Abel sums, Jacobi variety, and theta function in the scattering case, Invent. Math. 99(1990)483-544 
[23] N. Ercolani, M. Forest, D.W. McLaughlin, and R. Montgomery, Hamiltonian Structure for the Modulation Equations of a sine-Gordon Wavetrain, Duke Mathematical Journal, $\mathbf{5 5}(1987) 949-983$

[24] N. Ercolani, M. Forest and D.W. McLaughlin, Notes on Melnikov integrals for models of the periodic driven pendulum chain, preprint (1989)

[25] N. Ercolani and M. Forest, The Geometry of Real Sine-Gordon Wavetrains, Commun. Math. Phys. 99(1985)1-49

[26] N. Ercolani and D.W. McLaughlin, Toward a Topological Classification of Integrable PDE's,in: ed. by T.Ratiu, Proc. of a Workshop on The Geometry of Hamiltonian Systems, MSRI Publications, Springer-Verlag 22(1991)111-130.

[27] H. Flaschka and D.W. McLaughlin, Cannonically Conjugate Variables for the Korteweg-de Vries Equation and the Toda Lattice with Periodic Boundary Conditions, Prog. Theor. Phys. 55(1976) 2

[28] H. Flaschka, D.W. McLaughlin and M.G. Forest, Multiphase Averaging and the Inverse Spectral Solution of the Korteweg-de Vries Equation, Comm. Pure Appl. Math., 33(1980) 739

[29] H. Flaschka, A.C. Newell and T. Ratiu, Kac-Moody Lie Algebras and Soliton Equations II, III, Physica D 9(1983) 300-332

[30] M.G. Forest and D.W. McLaughlin, Spectral theory for the periodic sine-Gordon equation:A concrete viewpoint, J. Math. Phys. 23(1982)1248-1277

[31] M.G. Forest and D.W. McLaughlin, Modulation of Sinh-Gordon and Sine-Gordon Wavetrains, Studies in Appl. Math. 68(1983)11-59

[32] V. Guillemin and S. Sternberg, Geometric Asymptotics, Math.Sutveys 14(AMS Providence,Rhode Island)(1977)

[33] V. Guillemin and S. Sternberg, The Gelfand-Cetlin system and the quantization of the complex Flag Manifolds, J. Funct. Anal. 52(1983)106-128

[34] A. Hasegawa and Y. Kodama, Guiding-center soliton in optical fibers, Optical Letters, 15(1990) 1443-1445

[35] H. Knörrer, Singular fibres of the momentum mapping for integrable Hamiltonian systems, J. Reine u. Ang. Math. 35567-107.

[36] P.D. Lax and C.D. Levermore, The small Dispersion Limit of the Korteweg - de Vries Equation, I, II, III, Comm. Pure Appl. Math. 36 (1983)253-290, 571-593, 809-830

[37] J.E. Marsden, R. Montgomery and T. Ratiu, Cartan-Hannay-Berry Phases and Symmetry, Contemporary Mathematics 97,279 (1989); see also Mem. AMS 436(1990)

[38] V.P.Maslov, Complex WKB-method in nonlinear equations, (Nauka, Moscow, 1977) 
[39] V.P.Maslov, Perturbation Theory and Asymptotic Methods (Moscow University, Moscow, 1965)

[40] H.P. McKean, Integrable Systems and Algebraic Curves (Lecture Notes in Mathematics, Springer-Verlag, Berlin,1979)

[41] H.P. McKean, Theta functions,solitons, and singular curves, in: ed. by C.I.Byrnes, PDE and Geometry, Proc. of Park City Conference (1977)237-254

[42] H.P. McKean and E. Trubowitz, Hill's operator and hyperelliptic function theory in the presence of infinitely many brunch points, Comm. Pure Appl. Math. 29(1976)143-226

[43] D.W. McLaughlin and E.A. Overman, Whiskered Tori for Integrable PDE's: Chaotic Behavior an Near Integrable PDE's. Surveys in Appl.Math.,1(1992) (to appear)

[44] R. Montgomery, The Connection whose Holonomy is the classical Adiabatic Angles of Hannay and Berry and Its Generalization to the Non-Integrable case, Comm. Math. Phys.120 (1988)269-294

[45] J. Moser, Integrable Hamiltonian Systems and Spectral Theory (Academia Nazionale dei Lincei,Fermi Lecture, Pisa, 1981)

[46] D. Mumford, Tata Lectures on Theta I and II, Progress in Math.28 and 43,Birkhauser, Boston (1983)

[47] S.P. Novikov and A.P. Veselov, Poisson brackets and complex tori, Proc.Steklov Inst.Math.,3(1985)53-65

[48] E. Previato, Hyperelliptic quasi-periodic and soliton solutions of the nonlinear Schrödinger equation, Duke Math.Journal 52(1985)2

[49] S.N.M. Ruijsenaars, Action-Angle Maps and Scattering Theory for some FiniteDimensional Integrable Systems I.The Pure Soliton Case, Commun. Math. Phys. 115(1988)127-165

[50] E.R. Tracy, Topics in nonlinear wave theory with applications. Ph.D. Thesis, University of Maryland, College Park (1984)

[51] S. Venakides, The Generation of Modulated Wavetrains in the Solution of the Korteweg-de Vries Equation, Comm. Pure and Appl. Math., 38(1985)883-909

[52] S. Venakides and T. Zhang, Periodic Limit of Inverse Scattering, preprint, Duke University (1991)

[53] Yu.M. Vorob'ev and S.U. Dobrohotov, Quasiclassical quantisation of the periodic Toda chain from the point of view of Lie algebras, Theor.and Math. Phys. 54(1983)312-314

[54] A. Weinstein, Connections of Berry and Hannay type for moving Lagrangian submanifolds, Adv. in Math., 82(1990) 133-159 